\title{
SOME RESULTS IN GEOMETRY OF HYPERSURFACES
}

\author{
By TAKASHI OKAYASU
}

\section{Introduction.}

In this paper we get several theorems about hypersurfaces in space forms.

In section 1 , we show that if $x: M^{n} \rightarrow E^{n+1}$ is an isometric immersion of an $n$-dimensional complete non-compact Riemannian manifold whose sectional curvatures are greater than or equal to 0 , then $x(M)$ is unbounded in $E^{n+1}$. We can prove this using Sacksteder theorem [12] which states that under the above condition $x(M)$ is the boundary of a convex body in $E^{n+1}$. But his proof is rather long and his theorem is more than what we need. do. Carmo and Lima [3] gave an independent proof of Sacksteder theorem, but it is also long. So we give a direct and easy proof using so-called Beltrami maps which are defined in do. Carmo and Warner [4].

In section 2, we show that if $x: M^{n} \rightarrow S^{n+1}(1)$ is an isometric immersion of an $n$-dimensional complete Riemannian manifold whose sectional curvatures are less than or equal to 1 and $n$ is greater than 3 , then $x(M)$ is totally geodesic. Ferus almost proved this result in [6], [7]. We consider higher codimensional cases.

All manifolds we consider in this paper are class $C^{\infty}$, connected and have dimensions greater than or equal to 2 . All immersions and vector fields are $C^{\infty}$.

The author would like to express his hearty thanks to Professor S. Tanno for constant encouragement and advice.

\section{Unboundedness of hypersurfaces.}

The Beltrami maps are defined in M. do Carmo and F. Warner [2], and their properties are discussed fully.

Let $\nu \in S^{n+1}(1)\left(\subset E^{n+2}\right)$, and let $H_{\nu}$ denote the open hemisphere of $S^{n+1}(1)$ centered at $\nu$. The Beltrami map $\beta_{\nu}$ is the diffeomorphism of $H_{\nu}$ onto the hyperplane $S_{\nu} \subset E^{n+2}$ tangent to $S^{n+1}(1)$ at $\nu$ obtained by central projection. We consider $S_{\nu}$ to be equipped with the canonical Riemannian structure induced from $E^{n+2}$. $\quad \beta_{\nu}$ map great spheres of the sphere onto planes of $S_{\nu}$, and vice versa. We call this Beltrami map as spherical Beltrami map.

Received March 26, 1985 


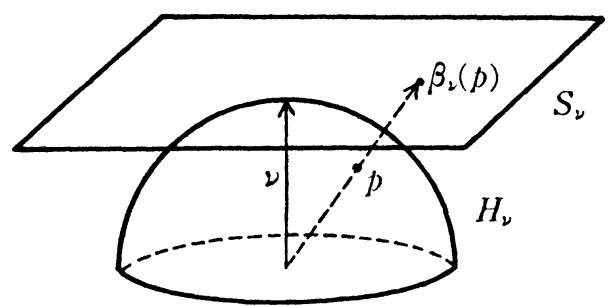

The following proposition is in [4].

Proposition 1. Let $\nu \in S^{n+1}(1)$, let $X \subset H_{\nu}$ be a hypersurface, and let $\tilde{X}$ denote the hypersurface $\beta_{\nu}(X)$ in $S_{\nu}$. Then $K_{X} \geqq 1$ everywhere if and only if $K_{\tilde{X}} \geqq 0$ everywhere.

Now we get the following.

THEOREM 1. Let $M^{n}$ be a complete non-compact Riemannian manifold, and suppose that there is a compact subset $C$ such that $K_{M} \geqq 0$ on $M \backslash C$. If $x: M^{n} \rightarrow$ $E^{n+1}$ is an isometric immersion, then $x(M)$ is unbounded in $E^{n+1}$.

Proof. Suppose $x(M)$ is bounded in $E^{n+1}$. We regard $E^{n+1}$ as $S_{\nu}$. We consider another Riemannian structure on $M$ with respect to which

$$
x: M^{n} \rightarrow S^{n+1}(1)
$$

is an isometric immersion. We denote $M$ with this Riemannian structure by $\tilde{M}$. It is easy to see that $\tilde{M}$ is complete. It follows from Proposition 1 that $K_{\tilde{M}} \geqq 1$ on $\tilde{M} \backslash C$. Using the same argument as in Bonnet theorem (cf. [2]), we conclude that $\tilde{M}$ is compact. This is a contradiction.

(q.e.d.)

We define hyperbolic Beltrami map $\beta_{a}$. Put

$$
\begin{aligned}
H^{n+1}(-1)= & \left\{\left(x^{1}, \cdots, x^{n+2}\right) \in \boldsymbol{R}^{n+2} ;\left(x^{1}\right)^{2}+\cdots+\left(x^{n+1}\right)^{2}\right. \\
& \left.-\left(x^{n+2}+1\right)^{2}=-1, \quad x^{n+2} \geqq 0\right\}
\end{aligned}
$$

where $\boldsymbol{R}^{n+2}$ is endowed with indefinite metric $\left(d x^{1}\right)^{2}+\cdots+\left(d x^{n+1}\right)^{2}-\left(d x^{n+2}\right)^{2}$. For $a>0$, we define the open cap $C_{a}$ as

$$
C_{a}=\left\{x \in H^{n+1}(-1) ; x^{n+2}<a\right\}
$$

Let $T_{a}$ be the $(n+1)$-dimensional plane which is perpendicular to the $x^{n+2}$-axis and contains $(0, \cdots, 0, a)$. The Beltrami map $\beta_{a}$ is the diffeomorphism of $C_{a}$ into the hyperplane $T_{a}$ obtained by the projection from the center $(0, \cdots, 0,-1)$. For this Beltrami map, we have a proposition similar to Proposition 1. 


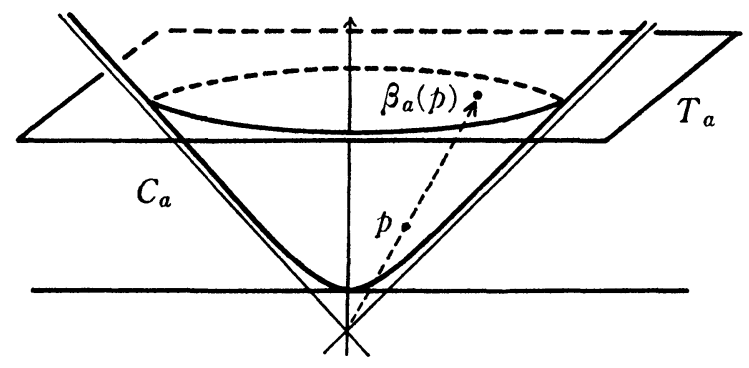

THEOREM 2. Let $M^{n}$ be a complete non-compact Riemannian manifold, and suppose there is a compact subset $C$ such that $K_{M} \geqq-1$ on $M \backslash C$. If $x: M^{n} \rightarrow$ $H^{n+1}(-1)$ is an isometric immersion, then $x(M)$ is unbounded in $H^{n+1}(-1)$.

Proof. Suppose $x(M)$ is bounded in $H^{n+1}(-1)$. We can assume $x(M)$ is contained in $C_{a}$. We define another Riemannian structure on $M$ with respect to which

$$
\beta_{a} \circ x: M^{n} \rightarrow T_{a}
$$

is an isometric immersion. We denote $M$ with this Riemannian structure by $\tilde{M}$. Then $\tilde{M}$ is complete and $K_{\tilde{M}} \geqq 0$ on $\tilde{M} \backslash C$, and $\tilde{M}$ is bounded in $E^{n+1}$. This contradicts theorem 1 .

(q. e. d.)

Next we turn to the negative curvature case.

Let $x: M^{n} \rightarrow N^{m}(c)$ be an isometric immersion of an $n$-dimensional Riemannian manifold $M$ in an $m$-dimensional Riemannian manifold $N$ with constant sectional curvature $c$. Let $h$ denote the second fundamental form. For $x \in M$, define

$$
T_{0}(x)=\left\{X \in T_{x} M ; h(X, Y)=0 \text { for all } Y \in T_{x} M\right\}
$$

$T_{0}(x)$ is called the space of relative nullity at $x$, and its dimension $\nu(x)$ is called the index of relative nullity at $x$. The minimal value $\nu_{0}$ of $\nu$ on $M$ is called the index of relative nullity of $M . \nu$ is upper-semicontinuous, and so the set $G$ where $\nu=\nu_{0}$ holds is open. The following theorem is well-known.

THEOREM 3 ([5], [9]). $\quad T_{0}$ is integrable on $G$, and its integral manifolds are totally geodesic submanifolds of $M$. They are totally geodesically immersed in $N^{m}(c)$ by $x$. If $M$ is complete, then the maximal integral manifolds of $T_{0} l_{G}$ are also complete.

Now we show the following.

THEOREM 4. Let $M^{n}$ be a non-compact complete n-dimensional Riemannian manifold, and suppose there is a compact subset $C$ such that $K_{M} \leqq 0$ on $M \backslash C$. If $x$ : $M^{n} \rightarrow E^{n+1}$ is an isometruc immersion and $n \geqq 3$, then $x(M)$ is unbounded in $E^{n+1}$. 
Proof. It follows from the curvature hypothesis that $\nu(x) \geqq n-2$ at every $x \in M \backslash C$. Put

$$
G_{0}=\{x \in M \backslash C ; \nu(x)=n-2\}
$$

Suppose $\bar{G}_{0}$ (the closure of $G_{0}$ ) is not compact. Since $G_{0}$ is open, we can choose $p_{k} \in G_{0}$ for any integer $k>0$ such that $d_{M}\left(p_{k}, C\right) \geqq k$. It follows from theorem 3 that the $(n-2)$-dimensional totally geodesic manifold in $M$ through $p_{k}$ can be extended so far as it meets $C$. This totally geodesic submanifold is also totally geodesic in $E^{n+1}$, and so $M$ is not bounded in $E^{n+1}$. If $\bar{G}_{0}$ is compact, put

$$
G_{1}=\left\{x \in M \backslash\left(C \cup \bar{G}_{0}\right) ; \nu(x)=n-1\right\}
$$

Suppose $\bar{G}_{1}$ is not compact. Since $G_{1}$ is open, the same argument as above holds good, and $M$ is not bounded in $E^{n+1}$. If $\bar{G}_{1}$ is compact, then $M \backslash\left(C \cup \bar{G}_{0} \cup \bar{G}_{1}\right)$ is non-compact and $\nu \equiv n$ there. So we see that $M$ is not bounded in $E^{n+1}$.

(q. e. d.)

We can show the following theorem in the same way.

THEOREM 5. Let $M^{n}$ be a non-compact complete n-dimensional Riemannian manifold, and suppose there is a compact subset $C$ such that $K_{M} \leqq-1$ on $M \backslash C$. If $x: M^{n} \rightarrow H^{n+1}(-1)$ is an isometric immersion and $n \geqq 3$, then $x(M)$ is unbounded in $H^{n+1}(-1)$.

\section{Submanifold with $K_{M} \leqq 1$ in $S^{n+p}(1)$.}

Consider the following question.

Let $x: M^{n} \rightarrow S^{n+p}(1)$ be an isometric immersion of a complete $n$-dimensional Riemannian manifold $M$ with $K_{M} \leqq 1$ in $S^{n+p}(1)$. Is $x(M)$ totally geodesic?

Of course if $p \geqq n-1$ the flat torus gives negative answer to this question. In low codimension we can give a partial positive answer. First we consider the case $p=1$.

THEOREM 6. If $n \geqq 4$ and $p=1$, then $x(M)$ is totally geodesic.

Proof. Assume $n \geqq 5$. It is easily proved that the index of relative nullity $\nu_{0} \geqq n-2$. If $\nu_{0} \geqq n-1$, then $K_{M} \equiv 1$ and according to O'Neill and Stiel [10], we can conclude that $x(M)$ is totally geodesic. So we suppose $\nu_{0}=n-2$. Choose a point $x \in M$ which satisfies $\nu(x)=n-2$. The maximal integral manifold $L_{1}$ of $T_{0}$ through $x$ is mapped to a $(n-2)$-dimensional great sphere in $S^{n+1}(1)$. Choose another point $y \in M$ which is not on $L_{1}$ and sufficiently near $x$. The maximal integral manifold $L_{2}$ of $T_{0}$ through $y$ is also mapped to a $(n-2)$-great sphere in $S^{n+1}(1)$. We consider $S^{n-1}(1)$ as the unit hypersphere in $E^{n+2}$. Since $L_{1}$ and 
$L_{2}$ do not intersect and $L_{1}, L_{2}$ are respectively on some $(n-1)$-planes through the origin in $E^{n+2}$,

$$
2(n-1) \leqq n+2
$$

holds; that is $n \leqq 4$, this is a contradiction.

If $n=4$, we need the following theorem due to Ferus [7].

Let $\rho(t)$ denote the largest integer such that the fibration

$$
V_{t, \rho(t)}^{\prime} \rightarrow V_{t, 1}^{\prime}
$$

of Stiefel manifolds has a global cross section (the points in $V_{t, r}^{\prime}$ are the ordered $r$-tuples of linearly independent vectors in $\boldsymbol{R}^{t}$ ). For every integer $n$ define $\nu_{n}$ to be the largest integer such that $\rho\left(n-\nu_{n}\right) \geqq \nu_{n}+1$.

THEOREM 7 ([7]). Let $M^{n}$ be an $n$-dimensional Riemannian manfold and $T_{0}$ a ע-dimensional, integrable distribution on $M^{n}$ with the following properties;

(1) the maximal integral manifolds of $T_{0}$ are totally geodesic and complete.

(2) the sectional curvature of $M$ has the same positive value $k$ on all planes spanned by tangent vectors $X, Y$ with $X \in T_{0}$ and $Y \in T_{0}^{\perp}$.

then $\nu>\nu_{n}$ implies $\nu=n$.

We finish the proof of theorem 6. As $\nu_{4}=0[7]$ and $\nu \geqq 2$, the conclusion follows.

(q. e. d.)

If $n=2,3$, there are counter-examples.

$$
\begin{aligned}
& n=2 \quad f: S^{1}(a) \times S^{1}(b) \rightarrow S^{3}(1) \quad\left(a^{2}+b^{2}=1\right) \\
& n=3 \quad\left\{\begin{array}{c}
2 x_{2}^{3}+3\left(x_{1}^{2}+x_{2}^{2}\right) x_{5}-6\left(x_{3}^{2}+x_{4}^{2}\right) x_{5} \\
+3 \sqrt{3}\left(x_{1}^{2}-x_{2}^{2}\right) x_{4}+3 \sqrt{3} x_{1} x_{2} x_{3}=2 \\
x_{1}^{2}+x_{2}^{2}+x_{3}^{2}+x_{4}^{2}+x_{5}^{2}=1 .
\end{array}\right.
\end{aligned}
$$

This is a homogeneous Riemannian manifold $S O(3) / \boldsymbol{Z}_{2} \times \boldsymbol{Z}_{2}$ and its principal curvatures are equal to $\sqrt{3}, 0,-\sqrt{3}[13]$.

We consider higher codimentional cases.

LEMma 1. Let $x: M^{n} \rightarrow N^{n+p}(c)$ be an isometric immersion of an n-dimensronal Riemannian manifold $M^{n}$ with $K_{M} \leqq c$ in $(n+p)$-dimensional Riemannian manifold $N^{n+p}(c)$ with $K_{N} \equiv C$. If the normal connection is flat and $n>2^{p}$, then the index of relative nullity $\nu_{0}$ satisfies $\nu_{0} \geqq n-2^{p}$.

Proof. If $p=1$, lemma is clear. Suppose $p=2$. Since the normal connection is flat, there exist orthonormal normal vector fields $\xi_{1}, \xi_{2}$ such that $A_{\alpha}(\alpha=1,2)$ is simultaneously diagonalizable where we write $A_{\alpha}=A_{\xi_{\alpha}}$, the second fundamental forms associated with $\xi_{\alpha}$. Let $\lambda_{a, \imath}(1 \leqq \imath \leqq n, 1 \leqq \alpha \leqq 2)$ be the eigenvalues 
of $A_{\alpha}$ corresponding to orthonormal eigenvectors $E_{\imath}$. Let $p, q, r$ be the numbers of positive, zero and negative $\lambda_{1, \imath}(1 \leqq i \leqq n)$. We may assume $p_{1} \geqq r_{1}$ (by the change of the sign of $\xi_{1}$ if neccessary). We may assume $\lambda_{1, \imath}>0\left(1 \leqq i \leqq p_{1}\right)$, and $\lambda_{1, j}=0\left(p_{1}+1 \leqq j \leqq p_{1}+q_{1}\right)$. We have

$$
p_{1}+q_{1} \geqq n / 2>2
$$

From Gauss equation and the curvature assumption, we have

$$
\lambda_{1, i} \cdot \lambda_{1, j}+\lambda_{2, i} \cdot \lambda_{2, \jmath} \leqq 0 \quad\left(1 \leqq i<j \leqq p_{1}+q_{1}\right) .
$$

Since $\lambda_{1, i} \geqq 0\left(1 \leqq i \leqq p_{1}+q_{1}\right)$

$$
\lambda_{2, i} \cdot \lambda_{2, \jmath} \leqq-\lambda_{1, i} \cdot \lambda_{1, \jmath} \leqq 0 \quad\left(1 \leqq \imath<j \leqq p_{1}+q_{1}\right) .
$$

Then the same arguement of the $p=1$ case applies, we have $p_{1}+q_{1}-2(>0)$ zeros in $\lambda_{2,3}\left(1 \leqq j \leqq p_{1}+q_{1}\right)$. If $p_{1} \leqq 1$, then $q_{1}=n-p_{1}-r_{1} \geqq n-2$. So we may assume $p_{1}>1$. It follows from (2) that the zeros are in $\lambda_{2,3}\left(p_{1}+1 \leqq \jmath \leqq p_{1}+q_{1}\right)$. So $q_{1} \geqq p_{1}+q_{1}-2$, that is $p_{1} \leqq 2$. Since $p_{1} \geqq r_{1}$, we have $r_{1} \leqq 2$. Hence

$$
q_{1}=n-p_{1}-r_{1} \geqq n-4>0 \text {. }
$$

This proves $p=2$ case. General case can be proved in the same way. (q. e. d.)

THEOREM 8. Let $x: M^{n} \rightarrow S^{n+p}(1)$ be an isometric immersion of an n-dimensional complete Riemannian manfold with $K_{M} \leqq 1$. If the normal connection is flat and $n \geqq 2^{p+1}$, then $x(M)$ is totally geodesic.

Proof. According to Ferus [7],

$$
\nu_{n} \leqq \frac{1}{2}(n-1) .
$$

On the other hand, from lemma, we have

$$
\nu_{0} \geqq n-2^{p} .
$$

The hypothesis $n \geqq 2^{p+1}$ implies

$$
\nu_{0} \geqq n-2^{p}>\frac{1}{2}(n-1) \geqq \nu_{n} .
$$

Thus it follows from theorem 7 that $\nu_{0}=n$, that is, $x(M)$ is totally geodesic.

(q. e. d.)

\section{Remarks.}

a) In theorem 1,2 higher codimensional cases don't hold. It is easy to construct counter-examples.

b) Using lemma 1, we can slightly extend theorem 3, 4 to higher codimensional cases. 
c) The case $n=2$ in theorem 4 , that is, the existance in $E^{3}$ of a complete bounded surface of non-positive curvature, is completely open. A possible example is constructed by Rozendorn [11] which has a denumerable number of isolated singular points. Note that in this example inf $K_{M}=-\infty$. This question is closely related to Jorge and Koutroufiotis [8].

d) When we almost finished this work, we found that Borisenko [1] had given positive answer to the question posed in section 2 under the condition that $M$ is compact and $p<-1 / 2+\sqrt{1 / 4+n / 2}$.

\section{REFERENCES}

[1] A. Borisenko, Complete $l$-dimensional surfaces of non-positive extrinsic curvature in a Riemannian space, Math. USSR Sb. 33 (1977), 485-499.

[ 2 [ J. Cheeger and D. Ebin, Comparison theorems in Riemannian Geometry, North Holland (1975).

[3] M.P. do CARMO AND E. Lima, Immersions of manifolds with non-negative sectional curvatures, Bol. Soc. Brasil Mat. 2 (1971), 9-22.

[4] M.P. do CARMO AND F.W. WARNAR, Rigidity and convexity of hypersurfaces in spheres, J. Diff. Geom. 4 (1970), 133-144.

[5] D. FERUS, On the completeness of nullity foliations, Michigan Math. J. 18 (1971), 61-64.

[6] D. FERUS, On the type number of hypersurfaces in spaces of constant curvature, Math. Ann. 187 (1970), 310-316.

[7] D. Ferus, Totally geodesic foliations, Math. Ann. 188 (1970), 313-316.

[8] L. Jorge And D. Koutroufiotis, An estimate for the curvature of bounded submanifolds. Amer. J. Math. 103 (1981), 711-725.

[9] R. Maltz, Isometric immersions into spaces of constant curvature, Illinois J. Math. 15 (1971), 490-502.

[10] B. O'NeILl AND E. STIEL, Isometric immersions of constant curvature manifolds, Michigan Math. J. 10 (1963), 335-339.

[11] E. R. ROzENDORN, Construction of bounded complete surface of nonpositive curvature, Usp. Math. Nauk. 16 (1961), 149-156. (in Russian)

[12] R. SACKSTEDER, On hypersurfaces with no negative sectional curvatures, Amer. J. Math. 82 (1960), 609-630.

[13] R. TAKAGI, Homogeneous hypersurfaces in a sphere with type number 2, Tōhoku Math. J. 23 (1971), 49-58.

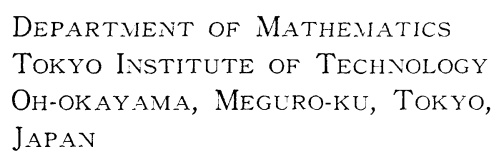

